\title{
Based on ODE and ARIMA Modeling for the population of China
}

\author{
Xiaohua Hu, Min Yu \\ School of Mathematics and Statistics, Hainan Normal University, Haikou, China \\ Email: 1241957415@qq.com
}

Received 2012

\begin{abstract}
The economic data usually can be also composed into a deterministic part and a random part. We establish ordinary differential equations (ODE) model for the deterministic part from a purely mathematical point of view, via the principle of integral and difference, establishing $\operatorname{ARIMA}(p, d, q)$ model for the random part, to combine the two established models to predict and control the original series, then we apply the method to study the population of China from 1978 to 2007, establishing the corresponding mathematical model, to obtain the forecast data of the population of China in 2008(1.3879503 billion), finally we make further stability analysis.
\end{abstract}

Keywords: Natural Asset; Financial Value; Neural Network

\section{Introduction}

A lot of time series (as economic data) can be regarded as a discretization of continuous-time process. if a time series $Y$ possesses biology background, we can establish a differential equations model for $Y$ in accordance with the growth rate, for example, suppose that constant $L$ is the growth limit of the variable $Y$, the rate of growth $d Y / d t$ is proportional to $Y$ or $L-Y$; suppose that the relative rate of growth $(d Y / d t) / Y$ is proportional to $(L-Y) / L$ or $\ln L-\ln Y$; some simple growth models can be established respectively, such as the Logistic model, Gompertz model[1] and so on, they are called the growth mechanism models.

In general, a time series $Q_{t}, t=0,1, \ldots, n$, can be decomposed into two parts[2]: a deterministic part $F_{t}$ and a random part

$h_{t}, t=0,1, \ldots, n$, or $Q_{t}=F_{t}+h_{t}, \quad t=0,1, \ldots, n$

(1)

where $F_{t}$ is usually described by the trend, seasonal and cyclic term, and $h_{t}$ is described by a more complex stochastic approach. In this paper, we ignore specific economic backgrounds of variable, from a purely mathematical point of view, to establish differential equations model for the discrete-time series $F_{t}$ on some conditions, while $h_{t}$ can be described by $\operatorname{ARIMA}(p, d, q)$ model[3].

\section{equations modeling}

Suppose that $y(t), 0 \leq t<+\infty$, is a continuous function, $F_{t}$ is regarded as the discretised value of $y(t)$. Set

$$
X(t)=\int_{0}^{t} y(t) d \approx \sum y(t) \Delta t
$$

$X^{\prime}(t)=y(t) \approx(X(t+\Delta t)-X(t)) / \Delta t$, as $\Delta t$ is small.

Now let's consider the discrete case (time series) and let $\Delta t=1$ (unit time). It follows that

$$
X(t) \approx \sum y(t), X^{\prime}(t)=y(t) \approx X(t+1)-X(t)
$$

Denoting that $y_{t}=y(t), X_{t}=X(t)$, if given a original time series $y_{t}=y(t), t=0,1, \ldots, n$, its cumulative sum series $X_{t}$ can be generated as $X_{t}=\sum_{k=0}^{t} y_{k}, t=0,1, \ldots, n$, so that, if we can find the relationship between the original series $y_{t}$ and its cumulative sum series $X_{t}$ as below,

$$
F\left(y_{t}, X_{t}, \varepsilon_{t}\right)=0, t=0,1, \ldots, n
$$

where $\varepsilon_{t}$ is a residual term, a random variable, satisfies $E\left(\varepsilon_{t}\right)=0, D\left(\varepsilon_{t}\right)=\sigma^{2}, \sigma>0$. We now view $y(t), X(t)$ as continuous, because of $X^{\prime}(t)=y(t)$, ignoring $\varepsilon_{t}$, corresponding to the one-order differential equation model can be established as follows.

$$
F\left(X(t), X^{\prime}(t)\right)=0 \quad \text { or } r \quad X^{\prime}(t)=f(X(t))
$$

We just need to solve out $X(t)$ from (2), so, $y_{t}=y(t)=X^{\prime}(t) \approx X(t+1)-X(t), y_{0}=y(0)=X(0)$ $t=1, \ldots, m . m \geq n$ (where $m$ is a positive integer).

\section{Principles or technology of differential}


In the same way, $X_{t}=X(t), t=0,1, \ldots, n$. is viewed as a new time series, we can generate its cumulative sum series $Z(t)=\sum X(t)$, or, the second cumulative sum of $y_{t}$, denote $Z_{t}=Z(t) \cdot Z^{\prime}(x)=X(t) \approx Z(t+1)-Z(t)$. we consider to establish the relationship of three series $y_{t}, X_{t}, Z_{t}$, if there exists the relationship as follows

$$
F\left(y_{t}, X_{t}, Z_{t}, \varepsilon_{t}\right)=0, t=0,1, \ldots, n
$$

where $\varepsilon_{t}$ is the same with the previously mentioned. We now view $y(t), X(t)$ as a continuous case, because of $Z^{\prime \prime}(t)=X^{\prime}(t)=y(t)$, ignoring $\varepsilon_{t}$, the two-order differential equation model can be established.

$$
\begin{aligned}
& F\left(Z^{\prime \prime}(t), Z^{\prime}(t), Z(t)\right)=0 \\
& \text { or } \quad Z^{\prime \prime}(t)=f\left(Z(t), Z^{\prime}(t)\right)
\end{aligned}
$$

we just need to solve out $Z(t)$ from (3), so,

$$
\begin{aligned}
& X_{t}=X(t)=Z^{\prime}(t) \approx Z(t+1)-Z(t), \\
& y_{t}=y(t)=X^{\prime}(t) \approx X(t+1)-X(t), \\
& y(0)=X(0)=Z(0), t=1, \ldots, m . m \geq n
\end{aligned} .
$$

In a general way, suppose that the original time series is $X_{0}(t)$. If $X_{0}(t)$ is not pure random data, its value's change on unit time is not random, or data has a trend. its the first cumulative sum series is $X_{1}(t), \ldots$, the $p$-th cumulative sum series is $X_{p}(t), t=1, \ldots, n$, where $p$ is a positive integer, if we can find the relationship of $X_{0}(t), X_{1}(t), \ldots, X_{p}(t)$ as below

$$
F\left(X_{0}(t), X_{1}(t), \ldots, X_{p}(t), \varepsilon_{t}\right)=0, t=0,1, \ldots, n
$$

where $\varepsilon_{t}$ is the same as previous, ignoring $\varepsilon_{t}$, the $p$-order differential equation model can be established.

$$
\begin{aligned}
& F\left(\frac{d^{p} X_{p}(t)}{d t^{p}}, \frac{d^{p-1} X_{p}(t)}{d t^{p-1}}, \ldots, \frac{d{ }_{p}(t)}{d t}, X_{p}(t)\right)=0 \\
& \text { or } \quad \frac{d^{p} X_{p}(t)}{d t^{p}}=f\left(\frac{d^{p-1} X_{p}(t)}{d t^{p-1}}, \ldots, \frac{d_{p}(t)}{d t}, X_{p}(t)\right)
\end{aligned}
$$

We just need to solve out $X_{p}(t)$, so

$$
\begin{gathered}
X_{i}(t)=X_{i+1}(t+1)-X_{i+1}(t), i=1, \ldots, p-1, t=1, \ldots, m . m \geq n \\
X_{0}(0)=X_{1}(0)=\ldots=X_{p}(0)
\end{gathered}
$$

It is usually difficult to find $F, f$, but, we can consider to establish the multiple linear (or nonlinear) regression model. Given a significance level $\alpha$ (for example, $\alpha=0.05$ ), if the significance test for the regression equation can be established, we can find the corresponding differential equations at $1-\alpha$ confidence level, which can explain the reasonable degree for the established differential equations. The adjusted R-squared $\left(R^{2}\right)$ or goodness-of-fit can describe the fitting degree of good or bad. We call $F=0$ the main model. for the residual series $\varepsilon_{t}$, we test that it is or isn't random by some test methods, if it isn't a pure random series(such as white-noise series[4]), it shows that there exists some valuable information hid in residual series, the informa- tion should be extracted out from the residual series by making use of the B-J method[5], at this time, we shall establish the model for residual series, It is called as the auxiliary model. Finally, we combine the main model and the auxiliary model to forecast.

\section{Empirical Analysis Application}

We study the Chinese population data[6] from 1978 to 2007 , the total number of sample observations is 21 , see

Table 1 Data of the population of China(unit:ten thousand) $\begin{array}{lllllllllll}1978 & 1980 & 1985 & 1990 & 1991 & 1992 & 1993 & 1994 & 1995 & 1996 & 1997\end{array}$ $\begin{array}{lllllllllll}9625 & 9870 & 1058 & 1143 & 1158 & 1171 & 1185 & 1198 & 1211 & 1223 & 1236\end{array}$ $\begin{array}{lllllllllll}9 & 5 & 51 & 33 & 23 & 71 & 17 & 50 & 21 & 89 & 26\end{array}$

1998199920002001200220032004200520062007

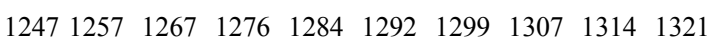

$\begin{array}{llllllllll}61 & 86 & 43 & 27 & 53 & 27 & 88 & 56 & 48 & 29\end{array}$

We use the sample data from 1978 to 2005 to model, leaving two sample data of 2006 and 2007 as a reference to assess the short-term forecast to see the accuracy of the established model.

\subsection{Analyse the Relationship Between the Original Series and its Cumulative Series}

Let the original sample time series be $y(t)=y_{t}, t=0,1, \ldots, 20$, the time range to model is from 1978 to 2005 .that is $t=0,1, \ldots, 18$, the first cumulative sum series of $y_{t}$ is $X_{t}=X(t)=X$, see fig.1,fig.2. We generate new series $Z_{t}, R_{t}$ via $y_{t}, X_{t}$ as follows

$$
Z_{t}=Z(t)=\frac{y_{t}}{X_{t}}, R_{t}=R(t)=\ln X(t)
$$

The scatter plot of $Z_{t}$ and $R_{t}$ is such as fig.3.We establish regression model below

$$
Z_{t}=c(1)+c(2) R_{t}+c(3) R_{t}^{2} .
$$

By making use of least-squares method and EViews6.0, it is easy to obtain the estimation value of parameters $c(1), c(2, \partial(3)$, see fig.4 and Tab.2.

$$
Z_{t}=22.19772-3.0877147 R_{t}+0.107731 R_{t}^{2}
$$

Table 2. Results of estimate and test (significance level $\alpha=\mathbf{0 . 0 5}$ )

$\begin{array}{ccccc}\text { Variable } & \text { Coefficient } & \text { Std. Error } & \text { t-Statistic } & \text { Prob. } \\ \text { c(1) } & 22.19772 & 1.334405 & 16.63491 & 0.0000 \\ \text { c(2) } & -3.0877147 & 0.202384 & -15.25674 & 0.0000 \\ \text { c(3) } & 0.107731 & 0.007644 & 14.09369 & 0.0000\end{array}$

The adjusted $R^{2}=0.986264$. The t-statistics of three regression coefficients $c(1), c(2, \partial(3)$ in (4) are respec- 
tively $16.64,-15.26,14.09$, their $\operatorname{Prob}=0.0000<0.05$,
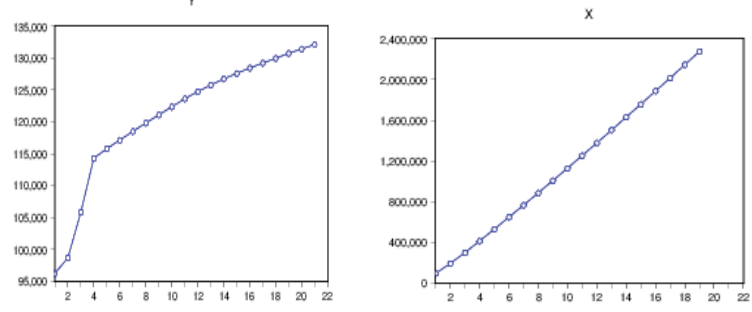

fig.1: the original time series $y$ fig.2: the cumulative sum series $X$

F-statistics $=647.2$. Prob $=0.0000<0.05$. the significant test for coefficients and the whole regression equation were resulted. We think that the Chinese population data from 1978 to 2005 can be described by (5) at $95 \%$ confidence level, Goodness-of-fit reaches $98.6 \%$. However, $\mathrm{DW}=1.317$ shows existence of autocorrelation in the residual series, It shows that there are still some valuable information not to be extracted out from the residual series, so we will establish the model for the residual series.
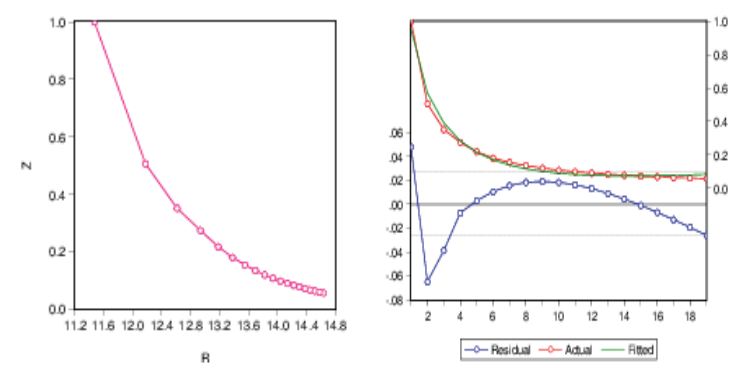

fig.3: the scatter plot of $R$ and $Z$ fig.4: the fitting and residuals plot of $R$ and $Z$

\subsection{Establish the Main Model-Differential Equ- ations Model}

It follows from (2),(4)and(5)that

$\frac{d X}{d t}=X^{\prime}(t)=\left[c(1)+c(2) \ln X(t)+c(3) \ln ^{2} X(t)\right] X(t)$

$$
\frac{d X}{d t}=X^{\prime}(t)=\left[22.198-3.088 \ln X(t)+0.108 \ln ^{2} X(t)\right] X(t)
$$

by formula[7], when $\sqrt{4 a c-b^{2}}>0$,

$$
\int \frac{d u}{a+b u+c u^{2}}=\frac{2}{\sqrt{4 a c-b^{2}}} \arctan \frac{2 c u+b}{\sqrt{4 a c-b^{2}}}+C,
$$

It is easy to obtain from (6)

$$
\frac{2}{\sqrt{4 c(1) c(3)-c^{2}(2)}} \arctan \frac{2 c(3) \ln X+c(2)}{\sqrt{4 c(1) c(3)-c^{2}(2)}}+C=t
$$

SO

$$
\begin{aligned}
X= & \exp \left\{\frac{1}{2 c(3)}\left[-c(2)+\sqrt{4 c(1) c(3)-c^{2}(2)}\right] \times\right. \\
& \left.\tan \left[\frac{1}{2} \sqrt{4 c(1) c(3)-c^{2}(2)}(t-C)\right]\right\}
\end{aligned}
$$

or

$$
\begin{aligned}
& X=\exp \left\{14.296+1.074 \tan \left(\frac{t}{8.624}+C 1\right)\right\} \\
& t=0, X(0)=96259, C 1=-69.519, \text { so } \\
& X=\exp \left(14.296+1.074 \tan \left(\frac{t}{8.624}-69.519\right)\right) \\
& \left.X^{\prime}=0.125 \sec ^{2}\left(\frac{t}{8.624}-69.519\right)\right) \exp ( \\
& \left.14.296+1.074 \tan \left(\frac{t}{8.624}-69.519\right)\right)
\end{aligned}
$$

\subsection{Establish the Auxiliary Model for Residual Series}

Let the residual series be $h_{t}$,we establish $\operatorname{ARIMA}(p, d, q)$ model for $h_{t}=h(t)$,make 1-order, 2-order difference for $h_{t}$ below

$$
\nabla h_{t}=h(t)-h(t-1)=h_{t}-h_{t-1}, \nabla^{2} h_{t}=\nabla h_{t}-\nabla h_{t-1}
$$

based on the analysis of the autocorrelation coefficients and partial autocorrelation coefficients of $h_{t}$ and 2-order difference of $h_{t}$. we take $p=q=d=2$, establish $\operatorname{ARMA}(2,2)$ model for $"{ }^{2} h_{t}$.

$$
\begin{aligned}
& \quad \nabla^{2} h_{t}=\varphi_{1} \nabla^{2} h_{t-1}+\varphi_{2} \nabla^{2} h_{t-2}+\varepsilon_{t}-\theta_{1} \varepsilon_{t-1}-\theta_{2} \varepsilon_{t-2} \\
& h_{t}=\left(2+\varphi_{1}\right) h_{t-1}+\left(\varphi_{2}-2 \varphi_{1}-1\right) h_{t-2}+\varphi_{1} h_{t-3} \\
& +\varphi_{2} h_{t-4}+\varepsilon_{t}-\theta_{1} \varepsilon_{t-1}-\theta_{2} \varepsilon_{t-2}
\end{aligned}
$$

this is a $\operatorname{ARIMA}\left(4,2,2\right.$; for $h_{t}$, where $\varepsilon_{t}$ is a white-noise series. the parameters in (10) are estimated by EViews6.0, $\hat{\varphi_{1}}=0.8436, \varphi_{2}=0.0328, \hat{\theta_{1}}=0, \theta_{2}=0.9992$.

$$
\begin{aligned}
& h_{t}=2.8436 h_{t-1}-2.6544 h_{t-2}+0.8436 h_{t-3} \\
& +0.0328 h_{t-4}+\varepsilon_{t}-0.9992 \varepsilon_{t-2}
\end{aligned}
$$

\subsection{Forecast Based on the Main and Auxiliary Model}

By (8) and (11), as $t=19,20,21, y=131190.5504$, 136191.0595, 138795.0589; $h_{t}=-0.007129,-0.0133$, -0.01979 , so, by (1), the predictive data of the population of China in 2006,2007 are 1.3119054,1.3619104 billion, respectively, however, the actual data are 1.31448, 1.32129 billion, respectively; the absolute errors are 2.58 , 40.62 ,respectively; the relative errors are $0.2 \%$, $3.1 \%$,respectively; It shows that the combination model $(1),(8)$ and (11) have a higher prediction accuracy, and the predictive data of the population of China in 2008 is 
1.3879503 billion. We also find that predictive value of the auxiliary model (11) is little impact on the total predictive value. the total predictive value mainly depends on the main model (8),or, mainly depends on the predictive value of the differential equations model (7). So, we can see that the short-term forecast accuracy is very high based on differential equations modeling for the time series on some condition.

We further consider the stability of equilibrium point of (6) or (7). Let

$\frac{d X}{d t}=\left[c(1)+c\left(2 \ln X(t)+c(3) \ln ^{2} X(t)\right] X(t)=f(X)=0\right.$
$X \neq 0,\left[c(1)+c(2) \ln X(t)+c(3) \ln ^{2} X(t)\right]=0 \quad, \quad$ when $c^{2}(2)-4 c(3) c(1)>0$, there are two real roots, denoted by $u_{1}, u_{2}$.there are two equilibrium points $X_{1}, X_{2}$, $X_{1}=\exp \left(u_{1}\right), X_{2}=\exp \left(u_{2}\right)$. on the other hand,

$$
f^{\prime}(X)=c(1)+c(2)+(c(1)+2 c(3)) \ln X+c(3) \ln ^{2} X,
$$

When $c^{2}(2)-4 c(3) c(1)>0$,

$$
\begin{aligned}
& f^{\prime}\left(X_{1}\right)=\sqrt{c^{2}(2)-4 c(3) c(1)}, \\
& f^{\prime}\left(X_{2}\right)=-\sqrt{c^{2}(2-4 c(3) c(1)} \cdot f^{\prime}\left(X_{1}\right)>0, f^{\prime}\left(X_{2}\right)<0
\end{aligned}
$$

The equilibrium point $X_{2}$ of (6) is stable, or, $t \rightarrow+\infty, X \rightarrow X_{2}$.the equilibrium point $X_{1}$ of (6) is unstable, or, $t \rightarrow+\infty, X \rightarrow+\infty$. so, we must control those factors that impact $c(1), c(2, \partial(3)$ in (6), such that $c^{2}(2)-4 c(3) c(1)>0$. otherwise, $t \rightarrow+\infty, X \rightarrow+\infty$ However, in fact, for model $(7), c^{2}(2)-4 c(3) c(1)<0$, it show that there is no equilibrium point in (7), it is ob- viously from (9), as $t / 8.624-69.519 \rightarrow \frac{\pi}{2}$, or $t \rightarrow 613, y \rightarrow+\infty$. it show that China's population will tend to infinity after 613 years, so, the model (7) is only suitable for short-term prediction.

\section{Acknowledgements}

Acknowledgements: the author is grateful to the anonymous referees for his helpful comments and suggestions.

\section{REFERENCES}

[1] Zhu Minhui. Fitting Gompertz Model and Logistic Model.J. Mathematics in Practice and Theory,2003;2:705-709.

[2] Peter J.Brockwell et al. Time series: theory and methods (2nd edn).China Higher Education Press Beijing and Springer-Verlag Berlin Heidelberg:Beijing,2001;75.

[3] Yi Danhui.Data analysis and Eviews application. China Statistics Press:Beijing,2002;66-70.

[4] Zhang Shiying.The financial time series analysis. Tsinghua University Press:Beijing,2008;90-93.

[5] Philip Hans Franses.Time Series Models for Business and Economic Forecasting[M].Beijing: Chinese People's University Press.2002.

[6] http//www.cpachn.org.cn/chinese/Teaching/Information.a sp?,2009-05-02.

[7] Tongji University Department of Applied Mathematics. Advanced Mathematics (5th edn), Beijing: Higher Education Press.2004. 